\title{
Hybrid optical pumping of optically dense alkali-metal vapor without quenching gas
}

\author{
M. V. Romalis \\ Department of Physics, Princeton University, Princeton, New Jersey 08544
}

\begin{abstract}
Optical pumping of an optically thick atomic vapor typically requires a quenching buffer gas, such as $\mathrm{N}_{2}$, to prevent radiation trapping of unpolarized photons which would depolarize the atoms. We show that optical pumping of a trace contamination of $\mathrm{Rb}$ present in $\mathrm{K}$ metal results in a 4.5 times higher polarization of $\mathrm{K}$ than direct optical pumping of $\mathrm{K}$ in the absence of $\mathrm{N}_{2}$. Such spin-exchange polarization transfer from optically-thin species is useful in a variety of areas, including spin-polarized nuclear scattering targets and electron beams, quantum-non-demolition spin measurements, and ultra-sensitive magnetometry.
\end{abstract}

Optical pumping is an extremely versatile technique that can by used to achieve nearly complete atomic polarization [1]. However, in an optically-thick medium radiation trapping imposes a limit on the maximum achievable polarization because spontaneously emitted photons do not have the same polarization and wavelength as the optical pumping light. Multiple reabsorption of these photons causes depolarization and for a high optical density completely prevents optical pumping [2]. It was realized soon after development of optical pumping that molecular buffer gases can be used to quench excited atomic states and prevent emission of spontaneous radiation. As a result, many applications of optical pumping, particularly for alkali-metal atoms, use $\mathrm{N}_{2}$ buffer gas to prevent radiation trapping [3]. However, there are several situations where the presence of 5-50 torr of $\mathrm{N}_{2}$ gas is detrimental. One example is polarization of $\mathrm{H}$ and D nuclear spins by spin exchange with alkali metals for use as nuclear targets in electron scattering experiments [4, 5]. A large magnetic field is used in this case to reduce the effect of radiation trapping [ 6 , 7] even though it also reduces the efficiency of spin-exchange, particularly for D [8]. Another example where the quenching gas is detrimental is for polarization of an electron beam using optically-pumped alkali-metal atoms [9, 10].

Spin-exchange collisions can be used to transfer spin polarization between different species, as was first demonstrated by Dehmelt [11]. Recently "hybrid" optical pumping using $\mathrm{Rb}$ and $\mathrm{K}$ atoms has been explored for spin-exchange optical pumping of ${ }^{3} \mathrm{He}$ gas [12, 13]. Optical pumping of $\mathrm{Rb}$ atoms with readily available lasers creates spin polarization in $\mathrm{K}$, which is then transferred to ${ }^{3} \mathrm{He}$ with a higher overall efficiency than by direct $\mathrm{Rb}-$ ${ }^{3} \mathrm{He}$ spin exchange [14]. Here we point out that hybrid optical pumping can be used in a wider set of circumstances when the optical density of one of the alkali-metal atoms is kept small. This allows optical pumping in the absence of $\mathrm{N}_{2}$ gas. In addition to the examples mentioned above, we point out that absence of $\mathrm{N}_{2}$ improves the effective optical depth in quantum non-demolition spin measurements using Faraday rotation [15].

Small optical density is also beneficial for spinexchange relaxation free (SERF) magnetometers [16]. In this case the limiting factor is not the presence of $\mathrm{N}_{2}$ gas, but the attenuation of the pumping beam. In steadystate operation the magnetometer has optimal sensitivity when the optical pumping rate is equal to the spin relaxation rate, a condition that cannot be maintained throughout an optically thick vapor. It is possible to use a far-detuned pump laser to reduce absorption, but this causes unwanted light shifts [17]. Optical pumping of a low-density alkali metal and Faraday rotation measurements on the high-density metal will achieve optimal sensitivity throughout the sensor.

We experimentally demonstrate spin polarization of optically dense $\mathrm{K}$ vapor without buffer gas by spin exchange with optically pumped $\mathrm{Rb}$. The spin polarization of $\mathrm{K}$ obtained in this way is a factor of 4.5 higher than by direct optical pumping of $\mathrm{K}$. Interestingly, the $\mathrm{Rb}$ metal was not intentionally introduced into the cell, but was present in the vapor at approximately $0.2 \%$ due to contamination of $\mathrm{K}$ metal. We also develop a density matrix model for optical pumping in the presence of high spinexchange rate but without any buffer gas and point out particular sensitivity to the circular polarization of the pumping light in this situation.

Consider two alkal-metal species undergoing spinexchange collisions, one much more abundant than the other. For definiteness we consider $\mathrm{K}$ and ${ }^{85} \mathrm{Rb}$, with a small Rb fraction $f=n_{R b} /\left(n_{R b}+n_{K}\right) \ll 1$. Rb atoms are being optically pumped with an average absorption rate $R$ for an unpolarized atom and both atoms are undergoing electron spin randomization collisions at a rate $\Gamma$. The thermally-averaged spin-exchange rate constant $\left\langle\sigma_{e x} v\right\rangle$ for different alkali-metal atoms as well as their mutual spin-exchange rates are nearly the same [18, 19]. Thus, the spin exchange rate of atom $i$ with atom $j$ is given by $X_{i j}=\left\langle\sigma_{e x} v\right\rangle n_{j}$ and the total spin-exchange rate $X=\left\langle\sigma_{e x} v\right\rangle\left(n_{R b}+n_{K}\right)$. If we make a simplifying assumption of fast electron spin randomization in the excited state, for example due to collisions with helium buffer gas, it is particularly easy to derive a simple equation for the spin polarization of $\mathrm{K}$ atoms due to optical pumping of $\mathrm{Rb}$ [20]. For $f \ll 1$ we get

$$
P_{K}=1 /[1+\Gamma(1 / R+1 / X) / f] .
$$




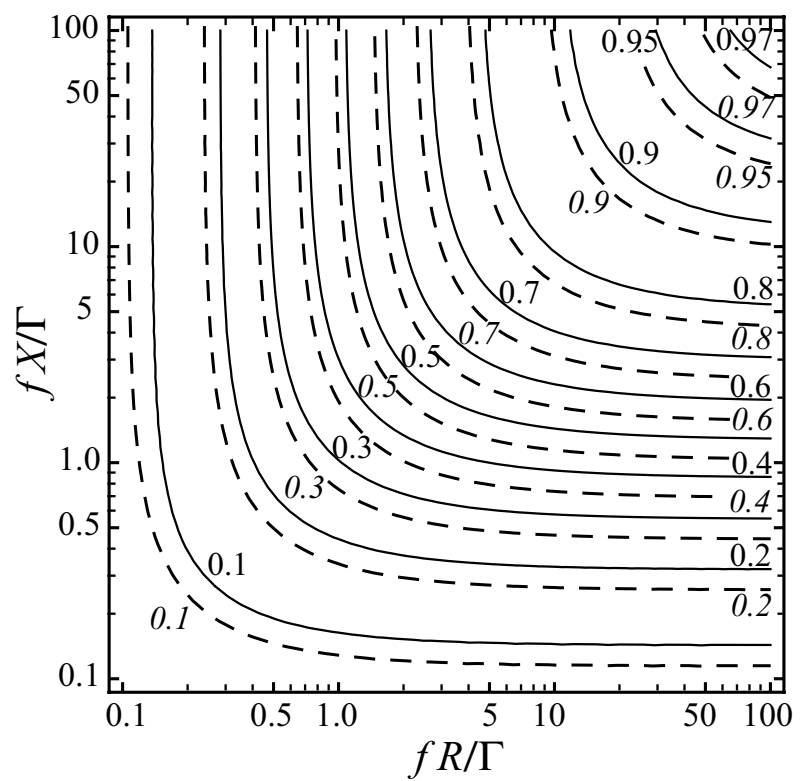

FIG. 1: Contour plot of maximum polarization of $\mathrm{K}$ as a function of the Rb pumping rate $R$, the spin exchange rate $X$, and the $\mathrm{Rb}$ fraction $f$. The solid lines and upright numbers correspond to the density matrix calculations while dashed lines and italic numbers correspond to Eq. (1)

Thus, two conditions need to be satisfied to achieve a high $\mathrm{K}$ polarization, $f R \gg \Gamma$ and $f X \gg \Gamma$. The first condition ensures that the overall input rate of angular momentum from the pump beam is sufficient to polarize all atoms and the second condition ensures that the spin exchange rate of $\mathrm{K}$ with $\mathrm{Rb}$ exceeds its own spin relaxation rate.

A more detailed analysis is necessary in the absence of buffer gas, when there is no electron randomization in the excited state and the depopulation optical pumping is determined by spontaneous emission. For this case we calculate the equilibrium density matrix in the presence of spin exchange, ground state electron spin relaxation, and optical pumping in vacuum. We assume the laser is tuned to the peak of $F=2 \rightarrow F^{\prime} \rightarrow 3 \mathrm{D} 1$ transition in ${ }^{85} \mathrm{Rb}$. Fig. 1 shows a contour plot of $P_{K}$ as a function of $f R / \Gamma$ and $f X / \Gamma$. The plot is made for $f=0.01$, but the results are virtually identical for $f=0.1-0.001$. One can see that Eq. (1), plotted with dashed lines, gives a good approximation to the full density matrix calculation.

In the absence of a buffer gas one typically uses an antirelaxation surface coating to reduce spin relaxation of alkali-metal atoms on cell walls [21]. The spin relaxation rate is then given by $\Gamma \simeq \bar{v} / l N_{e}$, where $\bar{v}$ is thermal velocity of atoms, $l$ is the characteristic dimension of the cell, determined by the volume/surface ratio, and $N_{e}$ is the number of bounces that the coating allows on average before electron spin relaxation. In order to avoid radiation trapping in optical pumping of $\mathrm{Rb}$ we need $\sigma_{0} n_{R b} l<1$. The $\mathrm{K}$ optical density $O D_{K}=\sigma_{0} n_{K} l$, where $\sigma_{0}$ is the

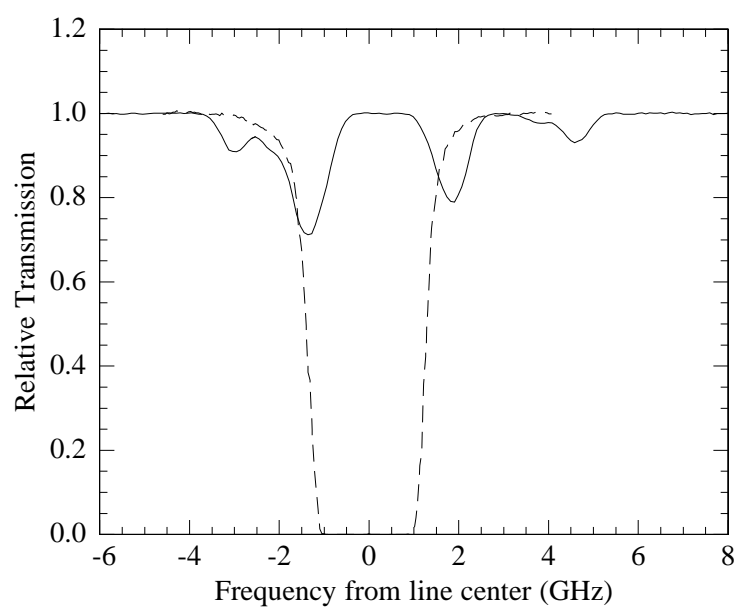

FIG. 2: Absorption spectrum of $\mathrm{K}$ (dashed line) and trace amounts of $\mathrm{Rb}$ (solid line) at $160^{\circ} \mathrm{C}$ in a $\mathrm{K}$ cell.

peak absorption cross-section, including the effects of Doppler broadening, which is similar for all alkali atoms. Thus, to avoid radiation trapping we need $f \simeq 1 / O D_{K}$. It follows that $f X / \Gamma=N_{e} \sigma_{e x} / \sigma_{0}$. The peak Doppler broadened absorption cross-section in alkali-metal atoms is about $\sigma_{0}=7 \times 10^{-12} \mathrm{~cm}^{2}$, while the spin exchange cross-section is $\sigma_{e x}=2 \times 10^{-14} \mathrm{~cm}^{2}$. Hence we need $N_{e} \gg 300$ in order to achieve high spin polarization. Paraffin has long been used as an anti-relaxation coating with operating temperature up to $50^{\circ} \mathrm{C}$. It typically allows about 10000 surface bounces before relaxation of the atomic polarization. The relaxation is predominantly due to electron spin randomization 22], so taking into account the nuclear slowing down factor it corresponds to $N_{e} \sim 1700$ for an alkali atom with $I=3 / 2$. More recently OTS coating with $N_{e}>300$ has been shown to operate at temperatures up to $170^{\circ} \mathrm{C}$ [23]. Alkane coatings recently reported in [24, 25] allow over $N_{e} \gg 10^{5}$ bounces and can operate up to $100^{\circ} \mathrm{C}$. A higher temperature results in a higher density of the more abundant alkali metal, which is advantageous for most applications.

We experimentally explore spin-exchange optical pumping in a 1.9 inch diameter evacuated cell with $\mathrm{K}$ metal and an OTS surface coating. Previously it was found that in the absence of $\mathrm{N}_{2}$ quenching the maximum $\mathrm{K}$ polarization achieved with optical pumping of $\mathrm{K}$ was limited to $2-3 \%$ [23]. It has been known anecdotally that commercial alkali-metal samples are often cross-contaminated, so a small impurity of Rb could be present in the cells. We have found that $\mathrm{Rb}$ vapor density was about $2 \times 10^{-3}$ of the $\mathrm{K}$ density and also observed trace amounts of $\mathrm{Cs}$ in the $\mathrm{K}$ cell. An absorption spectrum of $\mathrm{Rb}$ and $\mathrm{K}$ at $160^{\circ} \mathrm{C}$ is shown in Fig. 2. It can be seen that while $\mathrm{K}$ vapor is optically thick, the optical density of $\mathrm{Rb}$ is significantly less than 1 , ideal for optical pumping in the absence of quenching gas. 


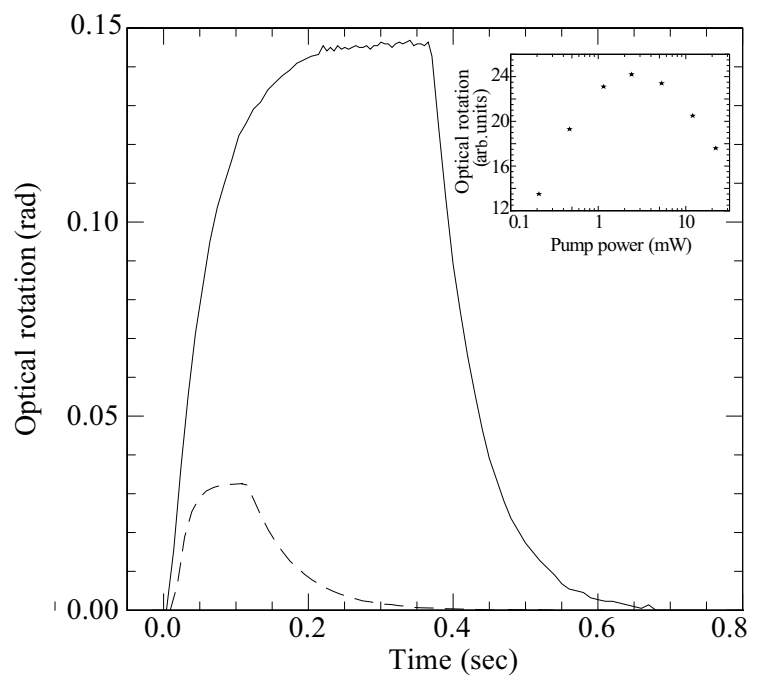

FIG. 3: Optical rotation transient in $\mathrm{K}$ vapor generated by optical pumping of optically dense $\mathrm{K}$ (dashed line) or trace amounts of $\mathrm{Rb}$ (solid line). The pump light is abruptly turned on and off. The inset shows the maximum polarization of $\mathrm{K}$ as a function of $\mathrm{K}$ pumping laser power.

Fig. 3 shows measurements of the $\mathrm{K}$ spin polarization with paramagnetic optical rotation of an off-resonant linearly polarized laser detuned by $51 \mathrm{GHz}$ from the $\mathrm{K}$ $\mathrm{D} 1$ line. The $\mathrm{K}$ vapor is optically pumped by a copropagating circularly polarized laser tuned to the $\mathrm{Rb}$ or to the K D1 line and parallel to a holding magnetic field. The maximum polarization obtained when pumping on $\mathrm{Rb}$ is about 4.5 times larger than when pumping directly on $\mathrm{K}$. The inset shows the power dependance of the maximum rotation signal as a function of $\mathrm{K}$ pump laser power, demonstrating the saturation and eventual decrease of the polarization due to radiation trapping. In contrast, for $\mathrm{Rb}$ pumping the $\mathrm{K}$ polarization continues to increase with available laser power. For the data in Fig. 3 the $\mathrm{K}$ density is determined from spin-exchange relaxation rate to be $n_{K}=9.2 \times 10^{12} \mathrm{~cm}^{-3}$. The optical rotation corresponds to $\mathrm{K}$ polarization obtained with $\mathrm{Rb}$ pumping of $13.7 \%$ vs. $3.0 \%$ for direct pumping on K. The time constant for the transient decay of spin polarization in the dark is $T_{1}=62 \mathrm{msec}$, which corresponds to $N_{e}=120$, taking into account nuclear slowing down factor for K. We find that both Eq. (1) and the density matrix model predict the $\mathrm{K}$ polarization with Rb pumping to be $10-15 \%$, in agreement with experimental measurements within uncertainties of the input parameters.

The dependence of the $\mathrm{K}$ spin-polarization on the $\mathrm{Rb}$ pump laser frequency is not trivial, as shown in Fig. 4. At a low optical pumping rate, the spectrum of $\mathrm{K}$ spin polarization, shown with solid triangles, resembles the $\mathrm{Rb}$ absorption spectrum. However, if the optical pumping rate is larger than the spin-exchange rate, the polarization exhibits a more complicated profile that is very sen-

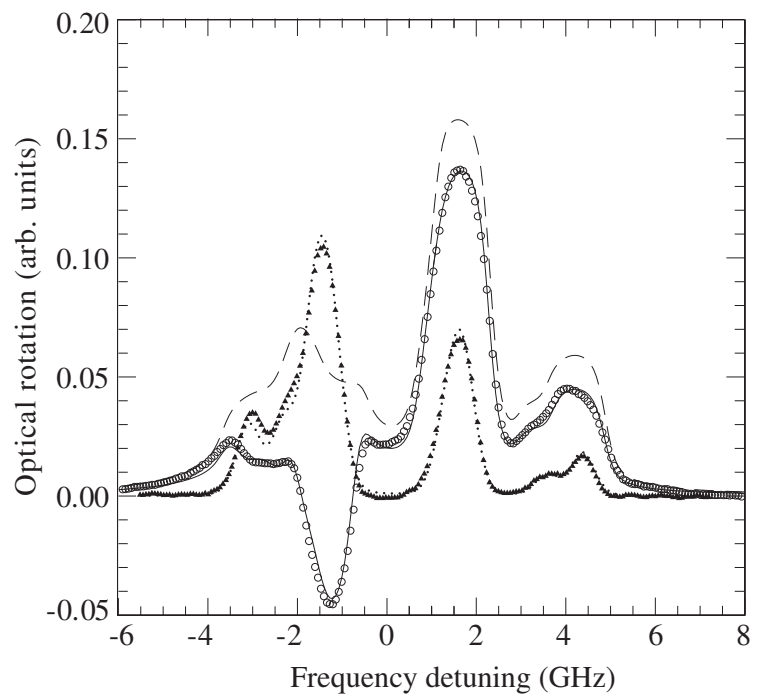

FIG. 4: Optical rotation in $\mathrm{K}$ vapor as a function of $\mathrm{Rb}$ pump light frequency. Open circles show K polarization measurements for $\mathrm{Rb}$ optical pumping rate much larger than the spin-exchange rate. Triangles show the $\mathrm{K}$ polarization for $\mathrm{Rb}$ pumping rate much smaller than the spin-exchange rate, with data scaled up by a factor of 14 . Note reversal of the polarization sign for pump light near $F=3{ }^{85} \mathrm{Rb}$ lines due to hyperfine optical pumping. The theoretical prediction for low pumping rate is shown with dots, for high pumping rate and perfect circular polarization with a dashed line and for $80 \%$ circular polarization with a solid line.

sitive to the degree of light polarization. As can be seen in Fig. 4, the measured polarization spectrum is very different from predictions for perfectly circularly polarized light, but follows closely the model assuming light polarization of $80 \%$. The polarization sensitivity is due to different degree of hyperfine optical pumping. For perfectly circularly polarized light $F=3, M=3$ state in ${ }^{85} \mathrm{Rb}$ is a dark state and $\mathrm{Rb}$ atoms can reach $100 \%$ polarization at high optical pumping intensity. However, if the light polarization is not perfectly circular, hyperfine pumping into $F=2$ state takes place when the optical pumping rate exceeds the spin exchange rate. This results in the reversal of the electron spin polarization of $\mathrm{Rb}$ as well as $\mathrm{K}$ atoms near $F=3{ }^{85} \mathrm{Rb}$ line.

Below we give two examples beyond those already explored in the literature where hybrid pumping with low optical density of one species is particularly useful. In quantum-non-demolition (QND) measurements using paramagnetic Faraday rotation [15] the intensity of the probe beam is usually limited by its absorption rate. In the regime of far detuning of the probe laser, the wing of the Voight absorption profile is dominated by the Lorentzian width $\Gamma_{L}$, which is the sum of the natural atomic decay rate and pressure broadening linewidth. The optical rotation angle is given by $\phi=n l r_{e} c f_{o s c}\left(\nu-\nu_{0}\right) /\left[\left(\nu-\nu_{0}\right)^{2}+\Gamma_{L}^{2}\right] / 2$, while the absorption cross-section is $\sigma=r_{e} c f_{o s c} \Gamma_{L} /\left[\left(\nu-\nu_{0}\right)^{2}+\Gamma_{L}^{2}\right]$. If the 
noise in measurements of Faraday rotation is dominated by photon shot noise, and spin relaxation of the atoms is limited by the probe beam photon absorption rate, one can show that the signal-to-noise ratio in one atomic spin relaxation time is equal to $\phi / \delta \phi=\sqrt{N_{a t} O D_{L}}$, where $O D_{L}=r_{e} c f_{\text {osc }} n l / \Gamma_{L}$. Hence, the atom spin can be readout with a SNR that exceeds atom shot noise $\sqrt{N_{a}}$ by a factor of $\sqrt{O D_{L}}$, thus allowing QND measurements that follow atomic spin evolution. The $O D_{L}$ that enters here corresponds to the Lorentzian linewidth, not to the actual observed optical depth on resonance, which is dominated by Doppler effect for hot atoms. In fact, for large probe laser detuning atom cooling does not offer any advantages in QND measurements. On the other hand, the presence of even a few torr of $\mathrm{N}_{2}$ gas, which is necessary for optical pumping of optically-dense vapor, dramatically increases the Lorentzian linewidth while having little effect on the overall absorption profile. For example, 10 torr of $\mathrm{N}_{2}$, which is the minimum typically required for quenching [10], increases the Lorentzian half-width in $\mathrm{Rb}$ by a factor of 39 from its value in vacuum $(3 \mathrm{MHz})$. In the absence of $\mathrm{N}_{2}$, the effective $O D_{L}$ for QND measurements is on the order of $5 \times 10^{4}$ for a $5 \mathrm{~cm}$ long cell with atom density of $10^{13} \mathrm{~cm}^{-3}$. Since one wins only relatively slowly with $O D$ in quantum measurements, such large optical densities are crucial to realizing a significant increase in sensitivity from quantum entanglement.

Different considerations apply for the use of hybrid optical pumping in spin-exchange relaxation free (SERF) magnetometers [16]. When the magnetometer is operated in steady-state regime the response to a magnetic field $B$ is given by $S=\gamma B R /(R+\Gamma)^{2}$. Hence the largest signal is obtained when $R=\Gamma$, corresponding to atomic spin polarization of $50 \%$. Since the atoms are not fully polarized, the circularly polarized pump laser is significantly absorbed. This limits the maximum density of the alkali metal so that the optical depth in the direction of the pump laser is $O D_{p} \sim 2$. Using hybrid optical pumping on a different alkali-metal with much smaller density eliminates this problem. For light alkalimetals, such as $\mathrm{K}$ and $\mathrm{Rb}$, the spin destruction crosssections are several orders of magnitude smaller than the spin-exchange cross-section. Therefore, spin polarization can be maintained with minor alkali-metal fractions of $f=10^{-2}-10^{-3}$, increasing operating alkali-metal density or the size of the cell by a large factor. We have explored this technique in a ${ }^{21} \mathrm{Ne}-\mathrm{Rb}$ co-magnetometer [26], using a Rb-K mixture with $f_{K}=0.005$ and optical pumping on $\mathrm{K}$. We obtained an order of magnitude higher polarization of ${ }^{21} \mathrm{Ne}$ than was possible using a single alkali-metal [27], opening new potential for sensitive nuclear spin gyroscopes [28].

In conclusion, we describe a simple technique for spin polarization of optically-thick alkali-metal atoms using spin-exchange with an optically-thin species. This approach, in combination with high quality anti-relaxation surface coatings, opens the possibility of creating very optically-dense spin-polarized alkali-metal vapors without any quenching gas. Such vapors can be used to transfer polarization to other species, such as atomic hydrogen or electrons. They are also useful for spin quantum nondemolition measurements. Even in the presence of buffer gas, hybrid optical pumping allows one to independently control absorption of optical pumping light, which is beneficial in ultra-sensitive atomic magnetometers and other optical pumping experiments.

We'd like to thank Scott Seltzer for fabrication of the alkali-metal cells and Ivanna Dimitrova for discussions. This work was supported by an ONR MURI award.

[1] W. Happer, Rev. Mod. Phys. 44, 169 (1972).

[2] D. Tupa and L.W. Anderson, Phys. Rev. A 362142 (1987).

[3] T.G. Walker and W. Happer, Rev. Mod. Phys. 69, 629 (1997).

[4] B. Clasie et al. Nucl. Instr. Meth. A 536, 260 (2005).

[5] B. Clasie et al., Phys. Rev. A 73020703 (2006).

[6] D. Tupa, L. W. Anderson, D. L. Huber, and J.E. Lawler, Phys. Rev. A, 33, 1045 (1986)

[7] L.W. Anderson and T. Walker, Nucl. Instr. Meth. A 316 , 123 (1992).

[8] M. Poelker et al. Phys. Rev. A 502450 (1994).

[9] H. Batelaan, A. S. Green, B. A. Hitt, and T. J. Gay, Phys. Rev. Lett. 82, 4216 (1999).

[10] M. A. Rosenberry, J. P. Reyes, D. Tupa, and T. J. Gay, Phys. Rev. A 75, 023401 (2007).

[11] H. G. Dehmelt, Phys. Rev. 109, 381 (1958).

[12] E. Babcock et al., Phys. Rev. Lett. 91, 123003 (2003).

[13] W. C. Chen, T. R. Gentile, T. G. Walker, and E. Babcock, Phys. Rev. A 75, 013416 (2007).

[14] A. B. Baranga et al. Phys. Rev. Lett. 80, 2801 (1998).

[15] Y. Takahashi et al., Phys. Rev. A 60, 4974 (1999).

[16] I.K. Kominis, T.W. Kornack, J.C. Allred, and M.V. Romalis, Nature 422, 596 (2003).

[17] V. Shah and M. V. Romalis, Phys. Rev. A 80, 013416 (2009).

[18] H. M. Gibbs and R. J. Hull, Phys. Rev. 153, 132 (1967).

[19] N. W. Ressler, R. H. Sands, and T. E. Stark, Phys. Rev. 184, 102 (1969).

[20] S. Appelt et al. Phys. Rev. A 58, 1412 (1998).

[21] H. G. Robinson, E. S. Ensberg, and H. G. Dehmelt, Bull. Am. Phys. Soc. 3, 9 (1958).

[22] D. Budker et al., Phys. Rev. A 71, 012903 (2005).

[23] S. J. Seltzer, M. V. Romalis, J. of Appl. Phys. 106, 114905 (2009).

[24] M. V. Balabas et al. Optics Express, 18,5825 (2010)

[25] M. V. Balabas, T. Karaulanov, M. P. Ledbetter, and D. Budker, Phys. Rev. Lett. 105, 070801 (2010).

[26] R. K. Ghosh and M. V. Romalis Phys. Rev. A 81, 043415 (2010).

[27] L.W. Cheuk, Senior Thesis, Princeton University (2010).

[28] T. W. Kornack, R. K. Ghosh, and M. V. Romalis, Phys. Rev. Lett. 95, 230801 (2005). 